\title{
Alcohol, wine and cardiovascular disease, two sides of the same coin
}

\author{
Ramon Estruch · Rosa Ma Lamuela-Raventós
}

Received: 23 February 2010/Accepted: 26 March 2010/Published online: 2 July 2010

(C) SIMI 2010

Excessive alcohol consumption is associated with increased morbidity and mortality, as well as with severe labour and traffic accidents. In fact, the dangers of alcohol intake have been with us throughout human history. The book of Genesis reported one of the first episodes demonstrating the dangers of alcohol. Upon leaving the Ark after the great flood Noah "drank wine and became drunk" and his sons had to protect him from embarrassment. Since then, the medical consequences of excessive alcohol consumption are well known and include acute intoxication, alcohol dependence, cirrhosis of the liver, pancreatitis, hypertension, stroke, dilated cardiomyopathy, cardiac arrhythmias and sudden death [1, 2].

On the other hand, human beings have drunk alcoholic beverages, especially wine, since ancient times and many believe in the beneficial effects of moderate wine intake. As an example, remains of a unique wine, shedeh, was found in the tomb of the pharaoh Tutankhamon to accompany him on his long trip to the "other life" [3]. However, the first modern evidence that there is a scientific link between alcohol consumption and cardiovascular disease was made in the early part of the last century, when it was observed that individuals that died from alcoholic cirrhosis had cleaner arteries than they ought to according to their age and lifestyle habits, suggesting that alcohol could exert a protective effect against atherosclerosis.

R. Estruch $(\square)$

Department of Internal Medicine, Hospital Clinic,

University of Barcelona, Barcelona, Spain

e-mail: RESTRUCH@clinic.ub.es

R. M. Lamuela-Raventós

Department of Nutrition and Food Sciences,

School of Pharmacy, University of Barcelona,

Barcelona, Spain
Since then, several epidemiological studies have suggested that light to moderate alcohol consumers have an increased survival and decreased risk for cardiovascular events than abstainers. A recent meta-analysis of 34 prospective cohort studies with more than 1 million subjects and almost 100,000 deaths by any cause showed a J-shaped association between self-reported alcohol consumption and mortality [4]. Current evidence suggests beneficial effects of moderate drinking on atherothrombotic events including coronary heart disease [5], ischemic stroke [6], peripheral arterial disease and, more recently, congestive heart failure [7]. Positive effects of moderate drinking on the quality of life, cognitive function, dementia and longevity have also been reported. These effects have been reported in a variety of patients, including diabetics, hypertensive subjects and patients with previous heart attack. As explained in the paper published by Di Castelnuovo et al. in this issue [8], the underlying mechanisms to explain the protective effects of moderate alcohol against coronary heart disease include an increase in high-density lipoprotein cholesterol (HDL-C), a decrease in platelet aggregation and coagulation effects, a reduction in the levels of fibrinogen and an increase in insulin sensitivity. This latter effect explains the reduced risk of diabetes in moderate drinkers. Additional beneficial effects of the intake of alcoholic fermented beverages against atherosclerosis have been attributed to their antioxidative and anti-inflammatory effects $[9,10]$ as well as their actions on vascular function.

However, although there are few doubts as to the beneficial effects of moderate drinking on cardiovascular system, a number of reports concerning alcohol and cancer have been published recently. According to the data of the Million Women study [11], even light to moderate levels of alcohol consumption were predictive of an increased risk of several common cancers, mainly those of the breast. Previous 
investigations on the association between alcohol and cancer have not been able to find any association between light alcohol intake and the diagnosis of cancer [12], whereas others have observed a negative association between light to moderate wine consumption and cancer [13]. In addition, several components of wine (e.g., resveratrol) have shown anti-cancer properties in experimental studies [14]. Thus, the issue of alcohol and cancer is absolutely open and new studies are needed. Since self-reported data on alcohol consumption in epidemiological studies may not be reliable, especially in women, clinical studies on the effects of alcohol on health should be based on specific and accurate biomakers of alcohol or wine consumption such as ethanol (or ethylenglicol) or resveratrol, respectively, in urine.

According to the above information, the recommended levels of "safe drinking" are up to two drinks (approximately $20 \mathrm{~g}$ ) on a given day for a man and up to one drink $(10 \mathrm{~g})$ per day for a non-pregnant woman. These different recommended daily doses of alcohol between genders are explained by the fact that women are more sensitive to the effects of alcohol on the body. The overall balance for young premenopausal women (who are at low risk for heart disease) would be unfavourable for drinking, but for older women (and for men) the risk of cancer would not outweigh the potential benefits of alcohol on heart disease.

Another important issue is the pattern of drinking and its main components, binge drinking and frequency of drinking. Research indicates that more frequent drinking, especially several days per week, is associated with more favourable outcomes than only occasional or weekly drinking. In addition, accumulated data suggest that low rates of coronary heart disease among the Mediterranean people are related to their traditional pattern of having wine with their meals every day [6].

Finally, with respect to the type of alcoholic beverages, international comparisons (French paradox) and some prospective study data suggest that wine is more protective against coronary heart disease than liquor or beer. In controlled clinical trials wine (ethanol plus polyphenols) exhibited significantly higher antioxidant and anti-inflammatory effects than gin (ethanol without polyphenols) [9, 10]. Possible non-alcohol beneficial components in wine may exert extra protection by wine. Studies in vitro and ex vivo indicated that polyphenols might exert some role in the prevention of certain vascular diseases, cancer, neurodegenerative diseases, diabetes and osteoporosis. Polyphenols may reduce atherosclerosis lowering blood pressure, inhibiting of oxidation of LDL particles and other favourable effects on cellular redox state, improving endothelial function, inhibiting platelet aggregation, reducing inflammation, and activating novel proteins that prevent cell death [14].

Therefore, as explained in detail in the review of Di Castelnuovo et al. in this issue, there is strong epidemiologic evidence of the protective effect of moderate alcohol consumption on cardiovascular disease. In addition, a large number of studies have shown a myriad of plausible mechanisms by which alcoholic beverages may exert their protective effect. However, in the era of evidence-based medicine, nutritional recommendations should be based on large-scale randomised intervention studies in which clinically relevant ("hard") end-points are evaluated. Up to now, no randomised controlled trial has ever been conducted to assess to what extent moderate alcohol consumption offers greater benefits than complete alcohol abstinence in the primary or secondary prevention of cardiovascular events. Without this level of evidence, many people may believe that the benefits of wine drinking may be explained by the fact that subjects, who prefer wine over beer or spirits tended to have relatively favourable characteristics, i.e. have a higher education and socioeconomic status and tended to eat a healthier diet, such as the Mediterranean diet [15].

Conflict of interest None.

\section{References}

1. Saitz R (2005) Unhealthy alcohol use. N Engl J Med 352:596607

2. Urbano-Márquez A, Estruch R, Navarro-López F, Grau JM, Mont Ll, Rubin E (1989) The effects of alcohol on skeletal and cardiac muscle. N Engl J Med 320:409-415

3. Guasch-Jané MR, Andrés-Lacueva C, Jáuregui O, LamuelaRaventós RM (2006) The origin of the ancient Egyptian drink Shedeh revealed using LC/MS/MS. J Archaeol Sci 33:98-101

4. Di Castelnuovo A, Costanzo S, Bagnardi V et al (2006) Alcohol dosing and total mortality in men and women: an updated metaanalysis of 34 prospective studies. Arch Intern Med 166:2437-2445

5. Rimm EB, Giovannuci EL, Willett WC et al (1991) Prospective study of alcohol consumption and risk of coronary disease in men. Lancet 338:464-468

6. Mukamal KJ, Ascherio A, Mittleman MA et al (2005) Alcohol and risk for ischemic stroke in men: the role of drinking patterns and usual beverage. Ann Intern Med 142:11-19

7. Djousse L, Gaziano JM (2008) Alcohol consumption and heart failure: a systematic review. Curr Atheroscler Rep 10:117-120

8. Di Castelnuovo A, Costanzo S, Donati MB, Iacoviello L, de Gaetano G (2010) Prevention of cardiovascular risk by moderate alcohol consumption: epidemiologic evidence and plausible mechanisms. Intern Emerg Med 5. doi:10.1007/s11739-0100346-0

9. Estruch R, Sacanella E, Mota F et al (2010) Moderate consumption of red wine, but not gin, decreases erythrocyte superoxide dismutase activity: a randomised cross-over trial. Nutr Metab Cardiovasc Dis (in press)

10. Estruch R, Sacanella E, Badia E et al (2004) Different effects of red wine and gin inflammatory biomarkers of atherosclerosis: a prospective randomized crossover trial. Atherosclerosis 175: $117-123$

11. Allen NE, Beral V, Cameron R et al (2009) Moderate alcohol intake and cancer incidence in women. $J$ Nat Cancer Inst 101:296-305 
12. Bessaoud F, Daurès JP (2008) Patterns of alcohol (especially wine) consumption and breast cancer risk: a case-control study among a population in Southern France. Ann Epidemiol 18: 467-475

13. Groenbaek M, Becker U, Johansen D et al (2000) Type of alcohol consumed and mortality from all causes, coronary heart disease and cancer. Ann Intern Med 133:411-419
14. Zamora-Ros R, Urpí-Sardà M, Lamuela-Raventós RM et al (2006) Diagnostic performance of urine resveratrol metabolites as a biomarker of moderate wine consumption. Clin Chem 52:1373-1380

15. McCann SE, Sempos C, Freudenheim JL et al (2003) Alcoholic beverage preference and characteristics of drinkers and nondrinkers in western New York (United States). Nutr Metab Cardiovasc Dis 13:2-11 